\title{
Theoretical Modeling and Analysis of Fiber-pull-out Behaviour from a Bonded Fibrous Matrix: The Elastic-bond Case
}

Ning Pan

Division of Textiles and Clothing, University of California, Davis, CA, U.S.A.

Received 30.9.1992 Accepted for publication 14.1.1993

A theory to characterize the single-fibre-pull-out behaviour of bonded fibrous systems is presented. Based on the shear-lag mechanism and the statistical-mean microstructural elements in the systems, the theory is developed for structures in which fibers are bonded with each other at contact points, and, as a result, the fiber-bonding with the matrix (the other fibers) is effected through the discrete points instead of a continuous interface. A theory aimed at one single bond is first derived, and it is then extended to the whole fiber by adding up the contributions from all bonds on this fiber into the result so as to predict a stepwise curve of the pull-out force and the embedded fiber length. This theory can also be used, combined with experimental results, to estimate the important bond properties, such as the bond shear strength $T_{b}$ and the shear modulus $G_{b}$. A parametric study is carried out and illustrated in this study to reveal the effects of the important factors involved during the fiber-pull-out process.

\section{NOTATION*}

The major symbols used in this paper are defined as follows.

$l_{f}, r_{f}$ and $D$ : Fiber length, radius, and diameter, respectively

$E_{f}, \sigma_{f}$ and $\sigma_{f u}:$ The fiber tensile modulus, the tensile stress in the fiber, and the fiber tensile strength, respectively

$V_{r}$ The fiber volume fraction of the system

$G_{b}$ and $\tau_{b}$ : The shear modulus and the shear strength of the bond between fibers, respectively

$\bar{n}$ and $\bar{n}_{f}$. The mean bonds on a fiber of length $l_{f}$ and the mean bonds per unit fiber length, respectively

$\bar{b}$ : The mean fiber length between two bond points

$\bar{b}_{b}$ and $w_{b}$ : The mean length and the mean width, respectively, of the bond

$t_{b}$ and $a_{1}$ : The equivalent mean thickness of the bond and the ratio of $t_{b}$ to the fiber radius

$r_{f}$, respectively

$b_{f}$. The mean free fiber length between two bonds

$m_{l}$ : The ratio of the mean free fiber length to the mean length

$p_{i}$ : The ratio of the mean bond length to the mean length

\section{INTRODUCTION}

The single-fiber-pull-out test in a fiber-composite study is one of several fundamental experimental approaches by which such issues as the nature of the interphase between the fiber and the matrix, the transfer of stress from matrix to fiber, and the strength and fracture of composites can be analyzed. The results from the fiber-pull-out experiments provide rich information in characterizing the interaction between fibers and the matrix. There have

* In this paper, the equations have been taken directly from the author's typescript instead of being set in the normal manner. The differential operator is set in italic type (d) instead of roman type (d), as is the usual practice. Subscripts to symbols are also set in italic type. 
therefore been numerous publications on this topic, and reference to a few representative papers $^{1-4}$ is provided in this paper.

However, the same topic does not seem to have attracted much interest from the researchers dealing with other categories of fibrous systems such as papers and nonwoven materials, in spite of the fact that, in these systems, fibers are bonded with each other by various means to form the porous but stable systems, and the fiber bond is also a crucial factor in determining almost every important property of the systems. Part of the reason may be the difficulty in actually carrying out the test: the fiber tensile strengths in these products are much lower, and the interfacial bonds between the fiber and the matrix (the adjacent fibers) are more complex; they are not even continuous as in composites.

Nevertheless, it has to be stressed that fiber-pull-out behaviour plays a key role in governing the fracture process and the strength of these products. After all, it is the interfacial bonds between fibers that generate sufficient adhesion between fibers and thus offer strength to these structures, which would otherwise be just loose fiber assemblies bound together through fiber entanglements and very weak cohesion between fibers. The bonds are therefore of particular importance in determining the mechanical performance and strength of the structures. Usually, the failure of these systems experiences three different mechanisms, i.e. fiber breakage, the bond-points failure, and fiber pull-out. Understanding of the fiber-pullout behaviour of these systems will hence enable us to predict the nature of the bonds between fibers so as to improve the quality of the products.

However, because of the significant differences between these fibrous systems and composites, the approaches, both analytical and experimental, developed for composite study are not readily applicable to these systems. A theory aimed specifically at such fibrous systems is therefore developed in this paper to predict the behaviour of the fiber-pull-out process in these systems. This theory can provide some insight into the interactions between fibers via bonds, characterize the bond properties, and reveal and estimate the important parameters involved. This theory should be helpful in promoting our understanding of the properties of such fibrous systems in providing information for the prediction of the mechanical properties and the various strengths of the fibrous structures.

\section{GEOMETRY OF THE BOND STRUCTURE AND ASSUMPTIONS}

A portion of a bonded fibrous structure is illustrated in Fig. 1(a). It is obvious that the properties of such a system are determined by the bonded areas, the free fiber segments between the bonding points, and the pores formed by fibers in the structure. Attention therefore has to be focused first on the characterization of these microstructural elements or. more specifically, on the investigation of the density and distribution of the bonding points and the relative proportions of bonded portions and free fiber segments on single fibers in the system. Because of the nature of the fibrous systems, these structural parameters vary with the fiber size (the length $l_{f}$, and the diameter $D=2 r_{f}$ ), the fiber amount, and the distributions of the fibers. We use the fiber aspect ratio $s(=l / D)$, the fiber volume fraction $V_{f}$ and the fiber orientation-density function to specify these system properties.

If we examine a typical and ideal structural element of the system in Fig. 1(b), it shows clearly that there are three basic segments, namely, the total length between the centers of the two adjacent bonds, the bond length, and the fiber length between the bonds. As stated above, the dimensions of all three structural features are statistical variables, depending on the sizes and the orientations of all fibers involved. It is therefore desirable that the statistical-mean values of them be used as shown in Fig. $1(b)$, where the mean fiber length between two bonds, designated as $\vec{b}$, consists of a bonded portion of mean length $\bar{b}_{b}$ and a free fiber segment of mean length $\bar{b}_{f}$ that is:

$$
\bar{b}=\bar{b}+\bar{b}_{b}
$$


Pan et $a l .{ }^{5}$ have defined previously two coefficients:

$$
m_{l}=\frac{\vec{b}}{\bar{b}}
$$

and

$$
p_{l}=\frac{\vec{b}}{\bar{b}}
$$

where

$$
m_{1}+\eta_{l}=1 \quad 0 \leq m_{1} \leq 1 \text { and } 0 \leq \eta \leq 1
$$

to represent the relative proportions of the bond portion and the free fiber length. Two extreme cases are when $m_{\mathrm{t}}=0$ and $p_{1}=1$, when fibers are totally bonded together, whereas $m_{1}=1$ and $p_{1}=0$ represent the case when the bond area does not exist. All these statistical parameters will be determined or explained in the later sections of this paper.

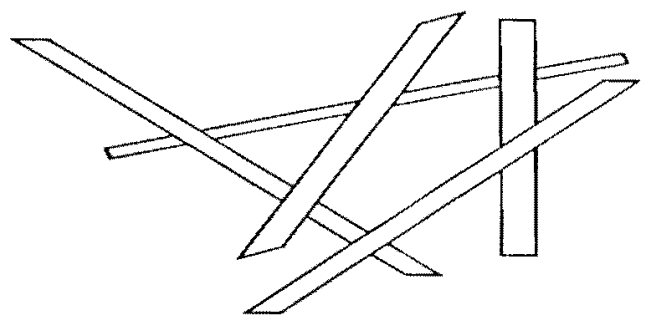

(a) A typical unit in a bonded fibrous system

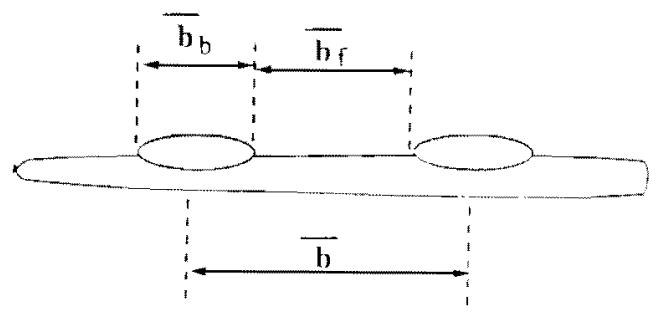

(b) The structural characteristics of a fiber elemcnt
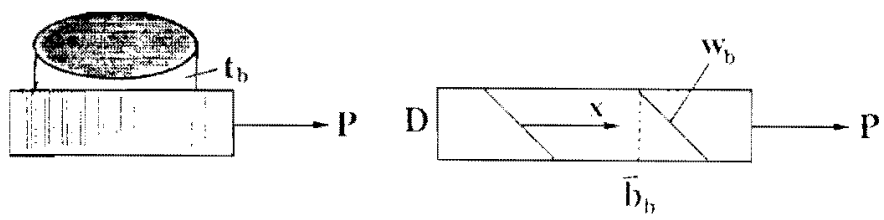

(c) The structural characteristics of a bond and the pull-out force

Fig. 1 The structural characteristics of a bonded fibrous system

Considering the nature of the fibrous systems in question and the fact that the model developed in the next section is in the nature of a shear-lag mechanism, the characteristics and assumptions associated with the systems and with this mechanism have to be considered or adopted, including first that, during the fiber-pull-out process, the extensional stress in the 
matrix (the surrounding bonded fibers) is negligible relative to that in the fiber. As a result, the distortion in the system due to fiber pull-out is small and excluded in the analysis. Secondly, the shear stress in the fiber is in turn negligible compared with that in the bond material, in other words, the shear deformation mainly takes place in the bond material between fibers.

One important parameter is therefore the thickness of the bond. Although, as shown in Fig. $1(c)$, the thickness of the bond material is not constant, for conciseness, we can always calculate an equivalent mean thickness, $t_{b}$, and further assume, without losing generality, that this thickness is proportional to the fiber radius $r_{f}$ i.e.:

$$
t_{b}=a_{1} r_{f}
$$

where $a_{1}$ is a constant to be determined.

Also excluded is the effect of the fiber Poisson's ratio during the pull-out process, so that the nature of the fiber-bond interaction will not change during the process.

It has to be emphasized that what are illustrated in Figures $1(b)$ and $1(c)$ are highly idealized situations, as are the assumptions associated with the present theory. For the cases where the fiber cross-section or the fiber-bond geometry is irregular, the present theory is still valid as long as the statistical mean values of these parameters are used.

\section{THEORETICAL ANALYSIS OF THE PULL-OUT PROCESS}

Most fiber-pull-out models on composites are variations of a model developed by Greszczuk ${ }^{2}$, which in turn is based on the well-known shear-lag theory. The theory proposed in this study also follows a similar theoretical frame but is significantly different from the previous ones owing to the differences of the present system.

From Fig. 1(c), showing the typical bond point, whose dimensions include the bond thickness $t_{b}$, length $\bar{b}_{b}$, and width $w_{b}$. we have:

$$
w_{b}=\sqrt{\left(\overrightarrow{b_{b}}-D\right)^{2}+D^{2}}
$$

If we ignore the stress variation over the fiber cross-section. we can set the central line of the bond width as the co-ordinate system $x$, as shown in Fig. 1(c). Suppose there is a pull-out force $P$ at one end of the fiber, which causes a tension $F$ on the fiber. The equilibrium of forces acting on an element $d x$ of the fiber requires:

$$
\frac{d F}{d x}=w_{b} \tau
$$

where $\tau$ is the shear stress developed in the bond. The tensile strain in the fiber is given by:

$$
\epsilon_{f}=\frac{d u}{d x}=\frac{F}{E_{f} \pi r_{f}^{2}}
$$

where $u$ is the displacement. The shear strain in the bond area is:

$$
\gamma=\frac{u}{t_{b}}
$$


and the shear stress is, by definition:

$$
\tau=\gamma G_{b}=\frac{u G_{b}}{t_{b}}
$$

Substituting Equation (10) into Equation (7) to eliminate $\tau$ and differentiating the resultant equation with respect to $x$, we obtain:

$$
\frac{d^{2} F}{d x^{2}}=p^{2} F
$$

where $\rho$ is a factor given by:

$$
\rho=\sqrt{\frac{G_{h}}{E_{f}} \frac{w_{b}}{\pi r_{f}^{2} t_{h}}}
$$

The solution of Equation (11) is:

$$
F=C_{1} \sinh \rho x+C_{2} \cosh \rho x
$$

where $C_{1}$ and $C_{2}$ are the integration constants and can be determined from the following boundary conditions:

$$
F=P \text { when } x=0 ; \quad F=0 \text { when } x=\overrightarrow{h_{b}}
$$

The final solution for the shear stress at $x$ of the bond point is:

$$
\tau=\frac{P \rho}{w_{b}}\left(\sinh \rho x-\operatorname{coth} \rho \bar{b}_{b} \cosh \rho x\right)
$$

The pull-out force $P$ can thus be obtained from this equation as a function of, among other parameters, the shear stress $\tau$.

The maximum pull-out force due to this single bond is dependent on the failure strength of the bond. There are two approaches to determine the failure strength of the interfacial bond, as was pointed out by $\mathrm{Kim}$ et al. ${ }^{6}$ : one is based on the maximum-shear-stress criterion such that bond failure will take place when the bond shear stress exceeds the bond shear strength; the other is based on the concept of fracture mechanics, where the debonded zone is considered as an interfacial crack, and its extension is dependent on the energy criterion's being satisfied. If we choose the former approach, the maximum pull-out force $P_{m}$ can be found when this shear stress reaches the shear strength of the bond $\tau_{b}$, i.e:

$$
\tau_{b}=\frac{P_{n e} \rho}{w_{b}}\left(\sinh \rho x-\operatorname{coth} \rho \overline{b_{b}} \cosh \rho x\right)
$$

and the maximum shear stress occurs at $x=0$, where:

$$
P_{m}=\frac{\tau_{b} w_{b}}{\rho} \tanh \rho \overline{b_{b}}
$$


If a fiber of length $l_{f}$ is totally embedded in the system, the total maximum pull-out force will be:

$$
P_{t m}=\vec{n} \frac{\tau_{b} w_{b}}{\rho} \tanh \rho \overline{b_{b}}
$$

For a fiber in the system with embedded length $L$, the total maximum pull-out force becomes:

$$
P_{l m}=\operatorname{Int}\left(n \vec{n}_{l} L\right) \frac{\tau_{b} w_{b}}{\rho} \tanh \rho \bar{b}_{b}
$$

where $\ln t^{\prime}()$ is the integer function which omits the fractional part of the result and yields only the integer so as to reflect the discrete bonds. It is shown clearly in the equation that, for a given embedded fiber length, the single-fibre-pull-out force is a function or an indicator of the number of bonds, the fiber thickness, and the fiber and bond properties.

On the other hand, whether an embedded fiber can be completely pulled-out from the matrix or break within it will depend on both the embedded length and the fiber tensile strength. Thus a critical fiber length $L=L_{c}$ is calculated according to the equilibrium on the fiber:

$$
P_{l m}=\sigma \sigma_{u} \pi \ddot{2}
$$

where $\sigma_{f u}$ is the fiber tensile strength.

\section{THE MICROSTRUCTURAL CHARACTERISTICS OF THE RANDOM FIBROUS SYSTEMS}

'To calculate the pull-out force by using the above equations, we need to know the parameters related to the microstructural characteristics of the system, such as the mean number of bonds per unit fiber length $\bar{n}_{i}$ and the mean length $\bar{b}_{b}$.

Although extensive work has been done on the microstructure analysis of paper by Kallmes and his colleagues ${ }^{7,8}$, by Page et al. ${ }^{9.10}$, and by Perkins et $a l^{11,12}$, and also on textile structures by van $\mathrm{Wyk}^{13}$, studying the mechanical properties of a fiber mass by looking into the microstructural units in the system, a far more general and elegant piece of research on this aspect was carried out by Komori and Makishima ${ }^{14}$, who have pioneered an approach dealing with the microstructural characterization of fibrous assemblies. However, during the application of this theory, some problems associated with it have been revealed ${ }^{15,16}$. Recently, there has been a study by the present author ${ }^{17}$ which shows that Komori and Makishima's prediction of the number of fiber contact points is too high, leading to a much shorter meanfibre-length value. Also in this study, the author has developed a modified theory by which the key variables in characterizing different fibrous systems have been predicted. These results are used here as a basis for the present work.

According to the present author's predictions ${ }^{17}$, for a three-dimensional fiber system where all fibers are oriented randomly with no preferred direction and bonded together to form the system, the mean number of bonds on an arbitrary fiber has been given as:

$$
\vec{n}=\frac{4 s V_{f}}{2+\pi V_{f}}
$$

the mean number of bonds per unit fiber length is:

$$
\bar{n}_{l}=\frac{4 V_{f}}{D\left(2+\pi V_{f}\right)}
$$


and the mean length between the two bonds is:

$$
\vec{b}=\frac{D\left(2+\pi V_{f}\right)}{4 V_{f}}
$$

In addition, the ratio of the mean free fiber length to the mean length between two bonds is:

$$
m_{l}=1-\frac{2 \pi V_{f}}{2+\pi V_{f}}
$$

and the ratio of bond length becomes

$$
p_{l}=1-m_{l}=\frac{2 \pi V_{f}}{2+\pi V_{f}}
$$

For a planar random-fiber network, the results will be different from those in a 3D system because the thickness of the system is restricted to twice the fiber diameter. The mean numbers of bonds on an arbitrary fiber now becomes from the earlier work ${ }^{17}$ :

$$
n=\frac{10 \pi s V_{f}}{\pi^{3}+16 \mathrm{AV}}
$$

where $A$ is a constant related only to the fiber dimensions as:

$$
A=\ln \left[\cot \left(\frac{\arcsin \left(\frac{D}{f f}\right)}{2}\right)\right]
$$

the mean number of bonds per unit fiber length is:

$$
\pi_{l}=\frac{16 \pi V_{f}}{D\left(\pi^{3}+16 A V_{f}\right)}
$$

and the mean length between the two bonds is:

$$
\bar{b}=\frac{D\left(\pi^{3}+16 A V_{f}\right)}{16 \pi V_{f}}
$$

Furthermore, the ratio of the mean free fiber length to the mean length between two bonds becomes:

$$
m_{l}=1-\frac{32 A V_{f}}{\pi^{3}+16 A V_{f}}
$$

and the ratio of bond lengths:

$$
p_{l}=\frac{32 A V_{j}}{\pi^{3}+16 A V_{f}}
$$


As stated above, because the thickness in a 2D system is limited to only twice the fiber diameter, this leads to differences in these microstructural characteristics. For example, the values of the mean length between two bonds $\bar{b}$ for $3 \mathrm{D}$ and $2 \mathrm{D}$ systems are not the same and the ratio of them,

$$
\frac{\bar{b}(3 D)}{\bar{b}(2 D)}=\frac{4 \pi\left(2+\pi V_{f}\right)}{\pi^{3}+16 A V_{f}}
$$

is related to both the fiber volume $V_{f}$ (the fiber amount) and the fiber size as represented by the factor $A$. These relations are illustrated in Fig. 2.

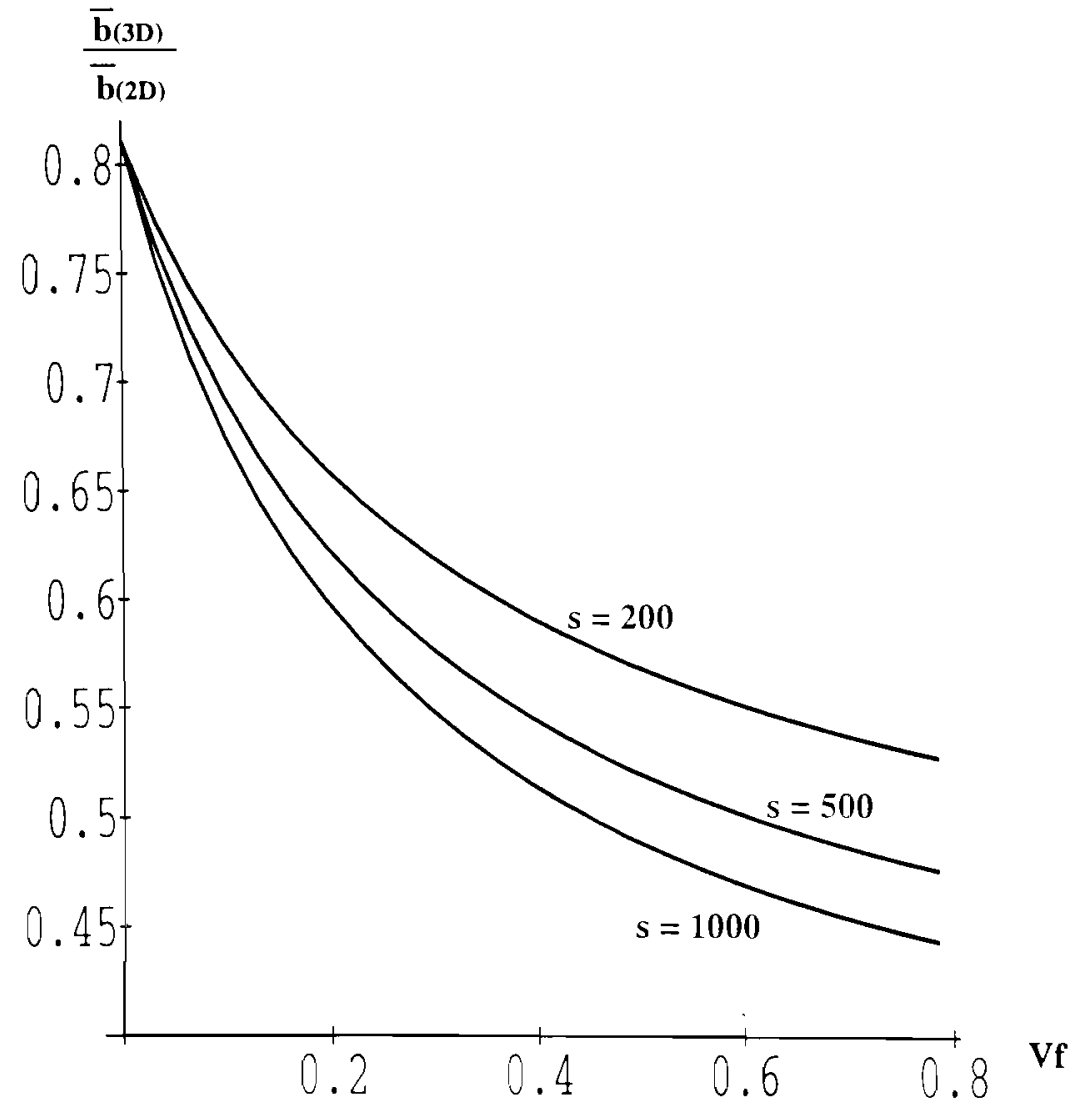

Fig. 2 Effects of $V_{1}$ and $s$ on the ratio of $\vec{b}$ values of 3D and 2D systems

From this figure, $\bar{b}$ for a $3 \mathrm{D}$ system is always smaller than the corresponding value for a $2 \mathrm{D}$ system. This difference will increase when either the fiber volume fraction or the fiber aspect ratio $s$ increases. On the other hand, the ratio of the bond lengths of the two systems

$$
\frac{\overline{b_{b}}(3 D)}{\overline{b_{b}}(2 D)}=\frac{\pi^{2}}{4 A}
$$

is independent of the fiber volume fraction and is determined only by the fiber size. Fig. 3 shows the relation of this ratio to the fiber aspect ratio. It is clear that, for the same fiber size, this value for a $3 \mathrm{D}$ system is always smaller than that for a $2 \mathrm{D}$ system. When the fiber becomes shorter or thicker, this difference reduces. 


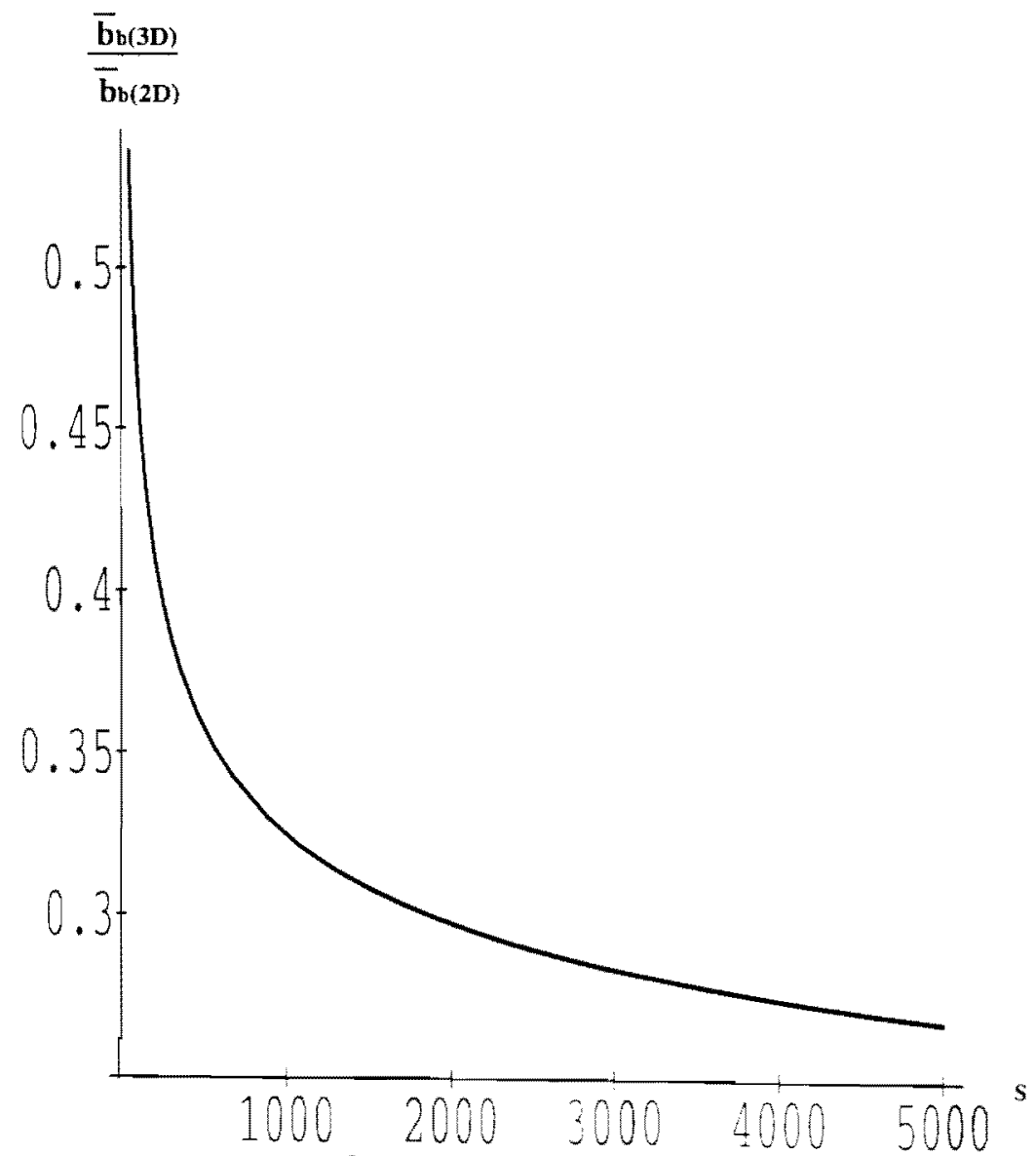

Fig. 3 Effect of $s$ on the ratio of $b_{n}$ values of $3 \mathrm{D}$ and $2 \mathrm{D}$ systems

In the following analysis, we focus only on the 3D system, and, because of the direct connections between the two systems as indicated in Equation (33), all conclusions in the following sections can be easily extended to a $2 D$ case. We thus have from Equations (23) and (25) the expression for the statistical mean bond length of a 3D system as:

$$
\overline{b_{b}}=p_{i} \vec{b}=\frac{\pi D}{2}
$$

\section{CALCULATIONS AND DISCUSSION}

It now has been shown that a bond such as the one in Fig. 1(c) can be characterized by the geometrical parameters, such as the bond thickness $t_{b}$, length $\bar{b}_{b}$, and width $w_{b}$, and the mechanical properties, including the bond shear modulus $G_{b}$ and the shear strength $\tau_{b}$. The parameters $\bar{b}_{b}$ and width $w_{b}$ can be directly calculated by using Equations (6) and (34) once the fiber diameter $D$ is given. The bond thickness $t_{b}$, on the other hand, has to be determined by relying on actual measurement for different systems or products.

The bond shear modulus $G_{b}$ and the shear strength $\tau_{b}$ are difficult to measure and seldom measured accurately. They can, however, be estimated with reasonable accuracy by using the 
theory. If we differentiate Equation (19) to give:

$$
\frac{d P_{l m}}{L}=\operatorname{int}\left(\bar{n}_{l}\right) \frac{r_{b} w_{b}}{\rho} \tanh \rho \overline{b_{b}}
$$

we find that the slope of the curve of the pull-out force plotted against the embedded fiber length is a constant. Note that, for a given system, $\bar{n}_{l}, w_{b}$, and $\bar{b}_{b}$ are considered to be known. Hence, on the basis of the experimental results, the two parameters $G_{b}$ (through the factor $\rho$ ) and $\tau_{b}$ can be determined from the results by using Equations (19) and (35).

For generality and ease of discussion, it is preferable to form some non-dimensional quantities in the resultant equations. First of all, since the quantity $\tau_{b} w_{b} / \rho$ has the units of force, Equation (17) therefore suggests that, because of the limited bond length $\bar{b}_{b}$, the pull-out force $P_{m}$ is discounted by an efficiency factor:

$$
\eta_{b}=\tanh \rho \overline{b_{b}}
$$

compared with the constant restraining force $\tau_{b} w_{b} / \rho$ exerted on the fiber, owing to the mechanism of stress transfer through the bond. When the bond length $\vec{b}_{b}$ approaches infinity, this discount will diminish as $\eta_{b} \rightarrow 1$.

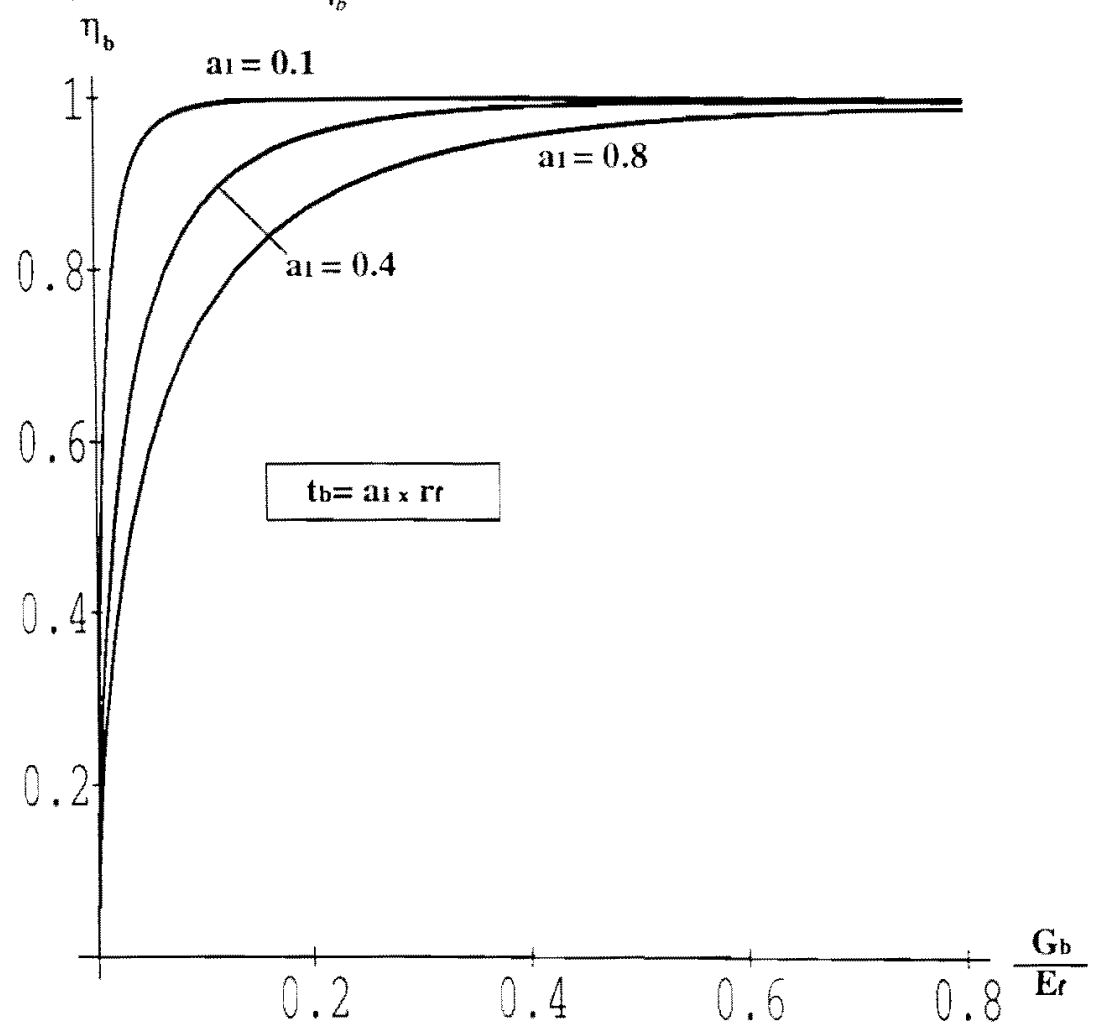

Fig. 4 Effects of $G_{b} / E_{f}$ and $a_{1}$ on the efficiency factor $\eta_{b}$

Fig. 4 depicts the effects of both the modulus ratio $G_{b} / E_{f}$ and the bond thickness $t_{b}$ on the efficiency factor $\eta_{b}$. In general, when the bond material is stiff in shear, or the fiber's Young's modulus is low, or the bond thickness is small, the bond will transmit the stress more efficiently, reflected by a high $\eta_{b}$ value.

Equation (19) can be rewritten in terms of the fiber tensile stress $\sigma_{f}$ according to: 


$$
P_{m}=\operatorname{Int}\left(n_{l} L\right) \frac{\tau_{1} w_{b}}{\rho} \eta_{k}=\pi r_{j}^{2} \sigma_{\rho}
$$

Defining a dimensionless quantity $\sigma_{f} / \tau_{b}$ in Equation (22), the equation becomes:

$$
\frac{\sigma_{f}}{\tau_{b}}=\operatorname{In} t\left(\bar{n}_{l} L\right) \frac{w_{b}}{\pi r_{f}^{2} \rho} \eta_{b}=\operatorname{Int}\left(\frac{2 V_{f}}{\left(2+\pi V_{f}\right)} \frac{L}{r_{f}}\right) \frac{w_{b}}{\pi r_{f}^{2} \rho} \eta_{b}
$$

This gives a non-dimensional or a more general relationship between the relative pull-out stress $\sigma_{f} / \tau_{b}$ and the relative embedded fiber length $L / r_{f}$ as well as the fiber- and bond-material properties.

Figures 5 and 6 are plotted by using this equation for a parametric study on the effects of the important factors involved. Fig. 5 corresponds to the fixed fiber volume fraction $V_{\mathrm{f}}=0.6$ and the bond thickness $t_{b}=a_{1} r_{f}$ where $a_{1}=0.8$. Because of the discrete distribution of all the bond points, the curves we have are not smooth but follow a stepwise path along with the fiber-length increment. It is shown in the figure that the pull-out force increases proportionally with the embedded fiber length. Moreover, when we increase the ratio $G_{b} / E_{f}$, meaning astiffer bond in shear or a lower fiber tensile modulus, for the same embedded fiber length, less force is required to pull it out of the matrix. The difference between Figures 5 and 6 is that, in Fig. 6 , the fiber volume fraction is allowed to change, the modulus ratio is fixed at $\mathrm{G}_{b} / \mathrm{E}_{f}=0.2$, and the bond thickness $t_{\mathrm{b}}$ is still equal to $=0.8 r_{f}$ This figure indicates that, for a given embedded fiber length, a high force will be required to pull the fiber out when there are more fibers in the system (i.e. a higher $V_{f}$ ).

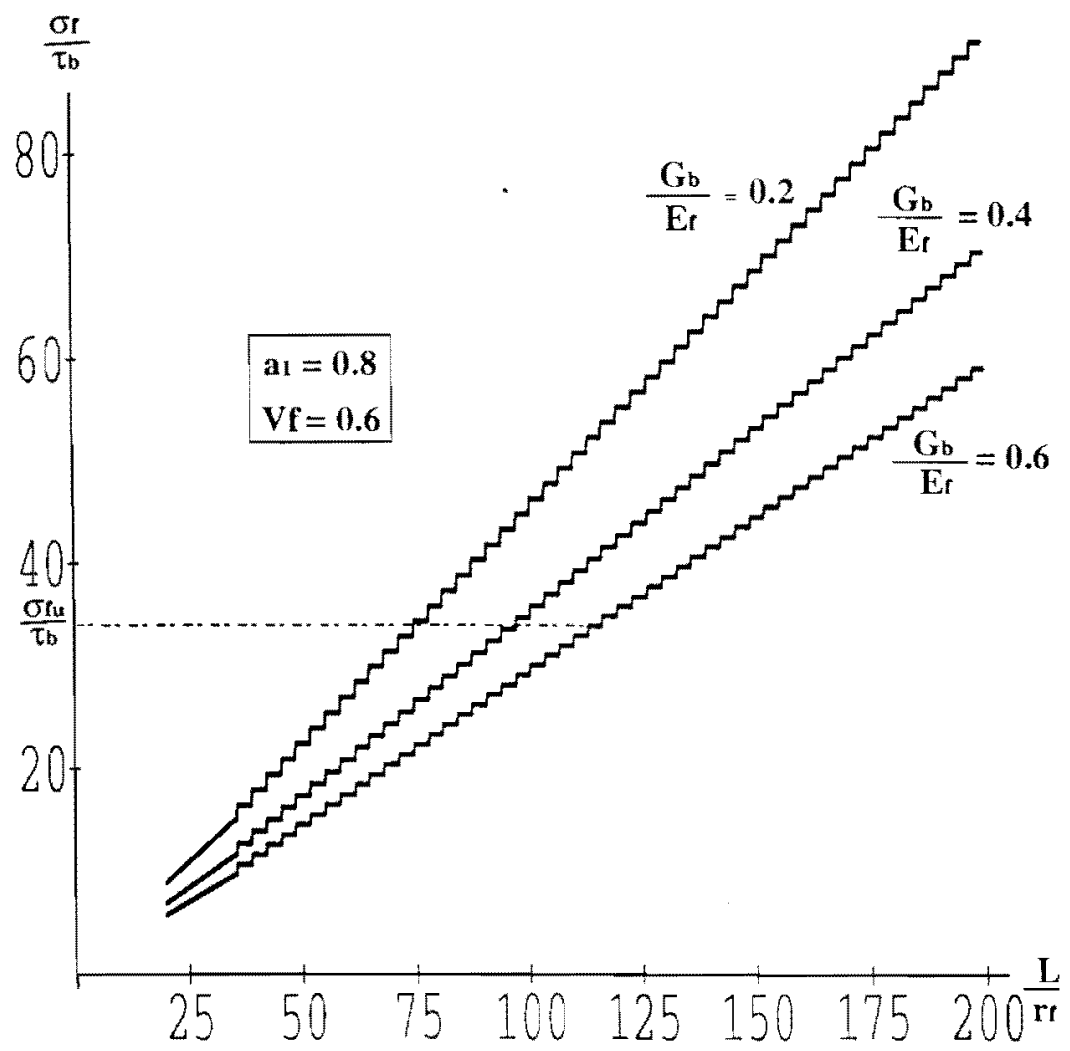

Fig. 5 Relationship between the relative pull-out stress and embedded fiber length at different $G_{a} / E_{\text {f }}$ levels 


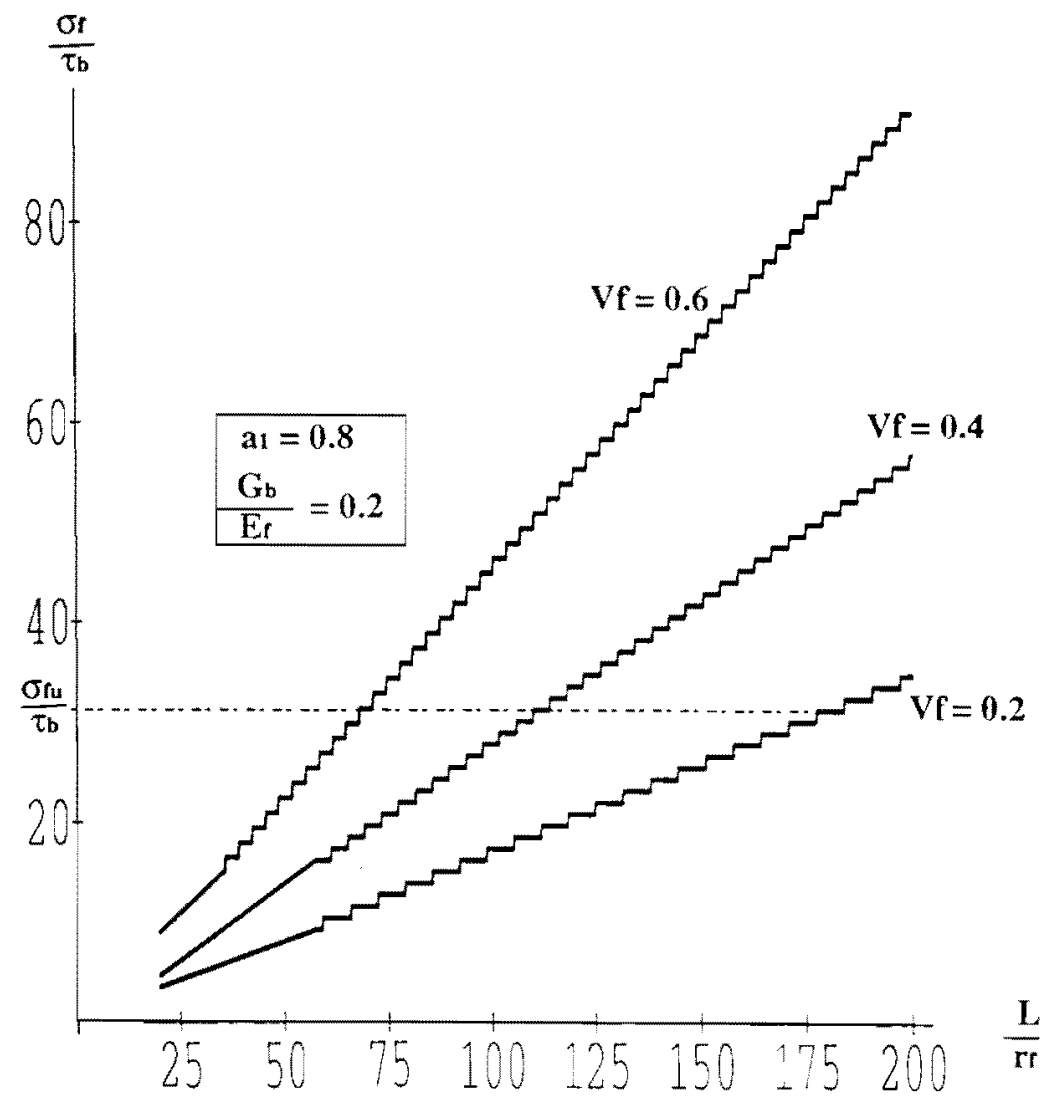

Fig. 6 Relationship between the relative pull out stress and embedded fiber length at different $V_{1}$ levels.

However, the actual pull-out force is limited to the value when the fiber tensile stress $\sigma_{\text {, }}$ reaches its tensile strength $\sigma_{f u}$, as is illustrated in the figures. This limit in turn defines the critical fiber length $L_{c}$ or the critical fiber aspect ratio $s_{c}$, beyond which the fiber will break inside the matrix rather than be pulled out. This critical fiber length $L$ or the critical fiber aspect ratio $s_{c}$ can also be expressed explicitly by a result derived from Equation (38) as:

$$
s_{c}=\frac{L_{c}}{D}=\operatorname{Int}\left(\frac{\left(2+\pi V_{f}\right)}{4 V_{f}} \frac{\pi r_{f}^{2} \rho}{w_{b} \eta_{b}} \frac{\sigma_{f u}}{\tau_{b}}\right)
$$

Since this equation is a result evolved from Equation (38), the effects of the modulus ratio $G_{b}$ f $E_{f}$ and the fiber volume fraction $V_{f}$ on the value of this critical fiber aspect $s_{\text {cc }}$ can be seen directly from Figures 5 and 6 if we replace the fiber tensile stress $\sigma_{f}$ by the tensile strength $\sigma_{f w}$ We also show the relationship between $s_{c}$ and the bond thickness $t_{b}=a_{r} r_{f}$ in Fig. 7. It indicates that the value of $s_{x}$ decreases with an increase in the $t_{b}$ value. This influence becomes more significant at higher values of the strength ratio $\sigma_{f u} / \tau_{b}$.

\section{CONCLUSION}

For a given fiber volume fraction of randomly distributed fibers, a 3D system corresponds to a shorter bond length than that of a 2D system, and the ratio between them is dependent on the fiber size. The bond thickness $t_{b}$ is a very crucial parameter in determining the mechanical behaviour of the system. 


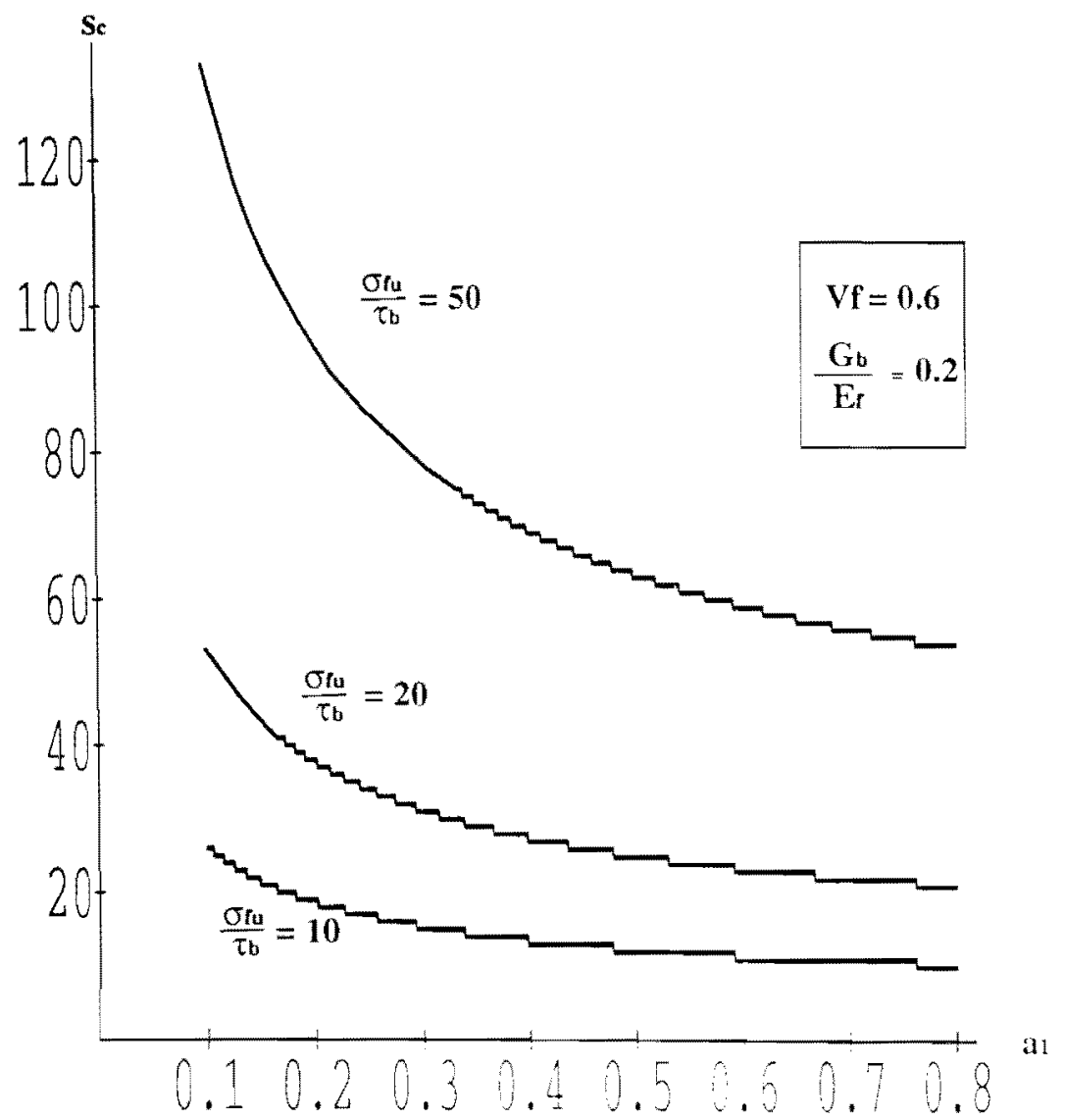

Fig. 7 Relationship between the critical fiber aspect ratios and the bond thickness factor $a$ at different $b$. levels

Statistically, the pull-out force in the fibrous systems dealt with in this study is proportional to the embedded fiber length. This is due to the constant mean bond length by which the stress transfer between the matrix and the fiber is achieved. The discontinuity of the distribution of the bonds on fibers is reflected on the curve in a stepwise form of the relation between pullout force and embedded fiber length. The pull-out force is also related to the fiber tensile modulus, the fiber diameter, the bond shear modulus, and the fiber volume fraction. Finally. the present theory can be used to estimate the bond shear strength $\tau_{k}$ and the shear modulus $G_{n}$

\section{REFERENCES}

P. Bartos. J. Mater. Sci., 1980, 15, 3122.

2.B. Greszczuk in 'Interfaces in Composites" (ASTM STP 4.52 ). A merican Sociely for Testing and Materals. Philadelphia, PA. U.S.A., 1969, p.42.

C.H. Hsueh. Mater. Sci. Engng, 1990, A123, 1.

* P. Lawrence. J. Mater. Sci., 1972, 7, 1 .

* N. Pan, J. Chen, Moon Seo, and 5. Backer. Papxr presented at IND h lundamental Rescarch Conference; Raleigh, NC, U.S.A., 21-22 July, 1992.

* J.K. Kim, C. Baillie, and Y.W. Mai. J. Mat'r Sci, 1992. 27, 314.3.

: O. Kallmes in Theory and Design of Wood and Fiber Composite Material tedited by B. A, Jaynel, Syracusc University Press, Syracuse, NY, U.S.A., 1972, p.157.

- O. Kallmes and H. Corte. Tappi, 1960, 43, 738 .

9 D.H. Page, R.S. Seth, and J.H. De Grace Tappi, 1979, 62, 99. 
10 D.H. Page, R.S. Seth, and J.H. De Grace. Tappi, 1980, 63,113.

$"$ R.W. Perkins and M.K. Ramasubramanian in 'Mechanics of Cellulosic and Polymeric Materials' (edited by R.W. Perkins), the American Society of Mechanical Engineers, New York, NY, U.S.A., 1989, p.23.

12 R.W.Perkins in 'Theory and Design of Wood and Fiber Composite Materials' (edited by B.A. Jayne). Syracuse University Press, Syracuse, NY, U.S.A., 1972, p.97.

${ }^{3}$ C.M. van Wyk. J. Text. Inst, 1946, 37, T282.

14 T. Komori and K. Makishima. Text. Res. J., 1977. 47, 13.

15 G.A. Carnaby and N. Pan. Text. Res. J., 1989, 59, 275.

is D.H. Lee, G.A. Camaby, A.J. Carr. and P.J. Moss. WRONZ Communication No. C113. 1990.

i) N. Pan. Text. Res. J, 1993, 63, 336. 